\title{
SUSY Quantum Hall Effect \\ on Non-Anti-Commutative Geometry ${ }^{\star}$
}

\author{
Kazuki HASEBE
}

Department of General Education, Takuma National College of Technology, Takuma-cho, Mitoyo-city, Kagawa 769-1192, Japan

E-mail: hasebe@dg.takuma-ct.ac.jp

Received October 01, 2007, in final form February 07, 2008; Published online February 22, 2008

Original article is available at http://www.emis.de/journals/SIGMA/2008/023/

\begin{abstract}
We review the recent developments of the SUSY quantum Hall effect [hep-th/0409230, hep-th/0411137, hep-th/0503162, hep-th/0606007, arXiv:0705.4527]. We introduce a SUSY formulation of the quantum Hall effect on supermanifolds. On each of supersphere and superplane, we investigate SUSY Landau problem and explicitly construct SUSY extensions of Laughlin wavefunction and topological excitations. The non-anticommutative geometry naturally emerges in the lowest Landau level and brings particular physics to the SUSY quantum Hall effect. It is shown that SUSY provides a unified picture of the original Laughlin and Moore-Read states. Based on the charge-flux duality, we also develop a Chern-Simons effective field theory for the SUSY quantum Hall effect.
\end{abstract}

Key words: quantum hall effect; non-anti-commutative geometry; supersymmetry; Hopf map; Landau problem; Chern-Simons theory; charge-flux duality

2000 Mathematics Subject Classification: 17B70; 58B34; 81V70

\section{Introduction}

Quantum Hall effect (QHE) provides a rare physical set-up for the noncommutative geometry (NCG), where the center-of-mass coordinates of electron satisfy the NC algebra

$$
[X, Y]=i \ell_{B}^{2} .
$$

Phenomena observed in QHE are governed by NCG and manifest its peculiar properties [1]. Until recently, it was believed QHE could be formulated only in 2D space. However, a few years ago, a 4D generalization of the QHE was successfully formulated in [2]. The 4D QHE exhibits reasonable analogous physics observed in 2D QHE, such as incompressible quantum liquid, fractionally charged excitations, massless edge modes and etc. The appearance of the 4D QHE was a breakthrough for sequent innovational progress of generalizations of the QHE. By many authors, the formulation of QHE has been quickly extended on various higher dimensional manifolds, such as complex projected spaces [3], fuzzy spheres [4, 5, 6], Bergman ball [7], a flag manifold $F_{2}$ [8] and $\theta$-deformed manifolds [9]. The developments of QHE have attracted many attentions from non-commutative geometry and matrix model researchers, since higher dimensional structures of NCG are physically realized in the set-up of the higher dimensional QHE. Indeed, the analyses of the higher dimensional QHE have provided deeper understandings of physical properties of NCG and matrix models ${ }^{1}$. Besides, $3 \mathrm{D}$ reduction of the $4 \mathrm{D}$ QHE gave a clue for the theoretical discovery of the spin Hall effect [11] in condensed matter physics.

\footnotetext{
*This paper is a contribution to the Proceedings of the Seventh International Conference "Symmetry in Nonlinear Mathematical Physics" (June 24-30, 2007, Kyiv, Ukraine). The full collection is available at http://www.emis.de/journals/SIGMA/symmetry2007.html

${ }^{1}$ For the higher dimensional developments of QHE and relations to fuzzy geometries and matrix models, interested readers may consult [10] as a good review.
} 
In this review, we report a new extension of QHE: a SUSY extension of the QHE, where particle carries fermionic center-of-mass degrees of freedom as well as bosonic ones: $(X, Y)$ and $\left(\Theta_{1}, \Theta_{2}\right)$. They satisfy the SUSY NC relations (non-commutative and non-anti-commutative relations):

$$
[X, Y]=i \ell_{B}^{2}, \quad\left\{\Theta_{1}, \Theta_{2}\right\}=\ell_{B}^{2}
$$

There are much motivations to explore the SUSY QHE. With the developments of string theory, it was found that the non-anti-commutative geometry is naturally realized on D-brane in graviphoton background $[12,13,14]$. The supermatrix models are constructed based on the super Lie group symmetries, and the non-anti-commutative geometry is embedded in supermatrix models by nature $[15,16]$. The SUSY QHE would provide a "physical" set-up which such string theory related models attempt to describe, and exhibit exotic features of the non-anti-commutative geometry in a most obvious way. Apart from possible applications to string theory, construction of the SUSY QHE contains many interesting subjects of its own right. QHE is deeply related to exotic mathematical and physical ideas: fuzzy geometry, Landau problem, Hopf fibration and topological field theory. As we supersymmetrize QHE, we inevitably encounter these structures. It is quite challenging to extend them in self-consistent SUSY frameworks, and interesting to see how they work.

Here, we mention several developments related to SUSY QHE and SUSY Landau problem. Algebraic and topological structures of the fuzzy supersphere are well examined in $[17,18]$. Field theory models on supersphere have already been proposed; a non-linear sigma model and a scalar field model were explored in [19] and [20], respectively. Numerical calculations on fuzzy spaces have also been carried out (see [21] as a review). The SUSY Landau problems on higher dimensional coset supermanifolds were developed in [22, 23]. Specifically in [22], the fuzzy super geometry on complex projective superspace $\mathbb{C} P^{n \mid m}=S U(n+1 \mid m) / U(n \mid m)$ was explored in detail. Planar SUSY Landau models were constructed in $[24,25]$ where the negative norm problem as well as its cure were discussed. (These works have several overlaps with our planar SUSY Landau problem developed in Subsection 5.2.) The spherical SUSY Landau problem with $\mathcal{N}=4$ SUSY was also investigated in [26]. Embedding of SUSY structure to QH matrix model has been explored in [27]. More recently, SUSY-based analysis was applied to edge excitations on the 5/2 filling $\mathrm{QH}$ state [28].

\section{Preliminaries}

A nice set-up for the QHE, needless to consider boundary effects, is given by Haldane [29] who formulated QHE on two-sphere with Dirac monopole at its center. We supersymmetrize Haldane's system by replacing bosonic sphere with supersphere, and Dirac monopole with supermonopole (see Table 1). The supersphere $S^{2 \mid 2}$ is a coset manifold taking the form of $O S p(1 \mid 2) / U(1)$. The adaptation of coset supermanifold has an advantage that the SUSY is automatically embedded by the coset construction. Here, we introduce basic mathematics needed to explore SUSY QHE.

\subsection{The $\operatorname{OSp}(1 \mid 2)$ super Lie algebra [30, 35]}

The $O S p(1 \mid 2)$ group is a super Lie group whose bosonic generators $L_{a}(a=x, y, z)$ and fermionic generators $L_{\alpha}\left(\alpha=\theta_{1}, \theta_{2}\right)$ satisfy the SUSY algebra:

$$
\left[L_{a}, L_{b}\right]=i \epsilon_{a b c} L_{c}, \quad\left[L_{a}, L_{\alpha}\right]=\frac{1}{2}\left(\sigma_{a}\right)_{\beta \alpha} L_{\beta}, \quad\left\{L_{\alpha}, L_{\beta}\right\}=\frac{1}{2}\left(C \sigma_{a}\right)_{\alpha \beta} L_{a},
$$


Table 1. The original Haldane's set-up and our SUSY extension.

\begin{tabular}{|c|c|c|}
\hline & The original Haldane's set-up & Our SUSY set-up \\
\hline Base manifold & $S^{2}=S U(2) / U(1)$ & $S^{2 \mid 2}=O S p(1 \mid 2) / U(1)$ \\
\hline Monopole & Dirac monopole & Supermonopole \\
\hline Hopf map & $S^{3} \rightarrow S^{2}$ & $S^{3 \mid 2} \rightarrow S^{2 \mid 2}$ \\
\hline Emergent fuzzy manifold & Fuzzy sphere & Fuzzy supersphere \\
\hline Many-body groundstate & $S U(2)$ invariant Laughlin & $O S p(1 \mid 2)$ invariant Laughlin \\
\hline
\end{tabular}

where $C$ denotes the charge conjugation matrix for $S U(2)$ group,

$$
C=\left(\begin{array}{cc}
0 & 1 \\
-1 & 0
\end{array}\right)
$$

The $O S p(1 \mid 2)$ algebra contains the $S U(2)$ subalgebra, and in the $S U(2)$ language, $L_{a}$ are $S U(2)$ vector and $L_{\alpha}$ are $S U(2)$ spinor. The differential operators that satisfy the $O S p(1 \mid 2)$ algebra are

$$
\begin{aligned}
& M_{a}=-i \epsilon_{a b c} x_{b} \partial_{c}+\frac{1}{2}\left(\sigma_{a}\right)_{\alpha \beta} \partial_{\beta}, \\
& M_{\alpha}=\frac{1}{2}\left(C \sigma_{a}\right)_{\alpha \beta} x_{a} \partial_{\beta}-\frac{1}{2} \theta_{\beta}\left(\sigma_{a}\right)_{\beta \alpha} \partial_{a} .
\end{aligned}
$$

The Casimir operator for the $O S p(1 \mid 2)$ group is given by $L_{a}^{2}+C_{\alpha \beta} L_{\alpha} L_{\beta}$ whose eigenvalue is $S\left(S+\frac{1}{2}\right)$ with integer or half-integer Casimir index $S$. The dimension of the irreducible representation specified by the Casimir index $S$ is $4 S+1$. In terms of $S U(2)$, the $O S p(1 \mid 2)$ irreducible representation $4 S+1$ is decomposed to $2 S+1 \oplus 2 S$, where $2 S+1$ and $2 S$ are $S U(2)$ irreducible representations with $S U(2)$ Casimir index $S$ and $S-1 / 2$, respectively. Since their $S U(2)$ spin quantum numbers are different by $1 / 2$, they are regarded as SUSY partners. The $O S p(1 \mid 2)$ matrices for the fundamental representation $(S=1 / 2)$ are given by the following $3 \times 3$ matrices:

$$
l_{a}=\frac{1}{2}\left(\begin{array}{cc}
\sigma_{a} & 0 \\
0 & 0
\end{array}\right), \quad l_{\alpha}=\frac{1}{2}\left(\begin{array}{cc}
0 & \tau_{\alpha} \\
-\left(C \tau_{\alpha}\right)^{t} & 0
\end{array}\right),
$$

where $\sigma_{a}$ are Pauli matrices, $C$ is the charge-conjugation matrix $(2.2)$, and $\tau_{1}=(1,0)^{t}, \tau_{2}=$ $(0,1)^{t}$. These matrices are super-hermitian, in the sense,

$$
l_{a}^{\ddagger}=l_{a}, \quad l_{\alpha}^{\ddagger}=C_{\alpha \beta} l_{\beta},
$$

where the super adjoint $\ddagger$ is defined by

$$
\left(\begin{array}{cc}
A & B \\
C & D
\end{array}\right)^{\ddagger}=\left(\begin{array}{cc}
A^{\dagger} & C^{\dagger} \\
-B^{\dagger} & D^{\dagger}
\end{array}\right)
$$

The complex representation matrices corresponding to $l_{a}$ and $l_{\alpha}$ are constructed as

$$
\tilde{l}_{a}=-l_{a}^{*}, \quad \tilde{l}_{\alpha}=C_{\alpha \beta} l_{\beta} .
$$

Short calculation shows that they actually satisfy the $O S p(1 \mid 2)$ algebra (2.1). It is important to note that the complex and the original representations are unitary equivalent,

$$
\tilde{l}_{a}=\mathcal{R}^{\dagger} l_{a} \mathcal{R}, \quad \tilde{l}_{\alpha}=\mathcal{R}^{\dagger} l_{\alpha} \mathcal{R},
$$


where the unitary matrix $\mathcal{R}$ is given by

$$
\mathcal{R}=\left(\begin{array}{ccc}
0 & 1 & 0 \\
-1 & 0 & 0 \\
0 & 0 & -1
\end{array}\right)
$$

Properties of $\mathcal{R}$ are summarized as

$$
\mathcal{R}^{t}=\mathcal{R}^{\dagger}=\mathcal{R}^{\ddagger}=\mathcal{R}^{-1}, \quad \mathcal{R}^{2}=\left(\mathcal{R}^{t}\right)^{2}=\left(\begin{array}{ccc}
-1 & 0 & 0 \\
0 & -1 & 0 \\
0 & 0 & 1
\end{array}\right) .
$$

$\mathcal{R}$ plays crucial roles in construction of SUSY Laughlin wavefunction and topological excitations as we shall see in Subsection 3.2.

\subsection{SUSY Hopf map and supermonopole [32, 33, 34]}

As is well known, the mathematical background of the Dirac monopole is given by the (1st) Hopf map (see for instance [31]). Similarly, the supermonopole ${ }^{2}$ is introduced as a SUSY extension of the Hopf bundle [32]. As the Hopf map is the mapping from $S^{3}$ to $S^{2}$, the SUSY Hopf map is the mapping from $S^{3 \mid 2}$ to $S^{2 \mid 2}$, which is explicitly

$$
\psi=\left(\begin{array}{c}
u \\
v \\
\eta
\end{array}\right) \rightarrow \frac{1}{R}\left(x_{a}, \theta_{\alpha}\right)=2 \psi^{\ddagger}\left(l_{a}, l_{\alpha}\right) \psi,
$$

or, with the complex representation,

$$
\psi=\left(\begin{array}{l}
u \\
v \\
\eta
\end{array}\right) \rightarrow \frac{1}{R}\left(x_{a}, \theta_{\alpha}\right)=-2 \psi^{t}\left(\tilde{l}_{a}, \tilde{l}_{\alpha}\right) \psi^{*}
$$

Here, $u$ and $v$ form Grassmann even $S U(2)$ spinor, while $\eta$ is a Grassmann odd $S U(2)$ singlet. The superadjoint $\ddagger$ is defined by $\psi^{\ddagger}=\left(u^{*}, v^{*},-\eta^{*}\right){ }^{3}$ With the constraint $\psi^{\ddagger} \psi=1, \psi$ is regarded as the coordinate on $S^{3 \mid 2}$, and $\left(x_{a}, \theta_{\alpha}\right)$ given by equation (2.5) (or equation (2.6)) automatically satisfy the relation that defines the supersphere $S^{2 \mid 2}$ with radius $R$,

$$
x_{a}^{2}+C_{\alpha \beta} \theta_{\alpha} \theta_{\beta}=R^{2} .
$$

$x_{a}$ and $\theta_{\alpha}$ represent bosonic and fermionic coordinates of supersphere, respectively. The SUSY Hopf spinor is simply a super coherent state;

$$
\frac{1}{R} l_{a} \psi \cdot x_{a}+\frac{1}{R} C_{\alpha \beta} l_{\alpha} \psi \cdot \theta_{\beta}=\frac{1}{2} \psi,
$$

or

$$
\frac{1}{R} \tilde{l}_{a} \psi^{*} \cdot x_{a}+\frac{1}{R} C_{\alpha \beta} \tilde{l}_{\alpha} \psi^{*} \cdot \theta_{\beta}=-\frac{1}{2} \psi^{*},
$$

\footnotetext{
${ }^{2}$ The supermonopole is usually referred to the graded monopole in literatures.

${ }^{3}$ It is noted that the symbol * does not denote the conventional complex conjugation but denotes the pseudoconjugation that acts to the Grassmann odd variables as $\left(\eta_{1} \eta_{2}\right)^{*}=\eta_{1}^{*} \eta_{2}^{*}$ and $\left(\eta^{*}\right)^{*}=-\eta$. See [30] for more details.
} 
as suggested by equation (2.5) or equation (2.6). The super Hopf spinor is explicitly represented as

$$
\psi=\frac{1}{\sqrt{2 R^{3}\left(R+x_{3}\right)}}\left(\begin{array}{c}
\left(R+x_{3}\right)\left(R-\frac{1}{4\left(R+x_{3}\right)} \theta C \theta\right) \\
\left(x_{1}+i x_{2}\right)\left(R+\frac{1}{4\left(R+x_{3}\right)} \theta C \theta\right) \\
\left(R+x_{3}\right) \theta_{1}+\left(x_{1}+i x_{2}\right) \theta_{2}
\end{array}\right) \cdot e^{i \chi},
$$

where the $U(1)$ phase $e^{i \chi}$ geometrically corresponds to $S^{1}$-fibre on $S^{2 \mid 2}$, and is canceled in the SUSY Hopf map (2.5) or (2.6). With the expression (2.8), the supermonopole gauge fields are explicitly calculated by the Berry phase formula:

$$
-i \psi^{\ddagger} d \psi=d x_{a} A_{a}+d \theta_{\alpha} A_{\alpha} .
$$

The results are

$$
\begin{aligned}
A_{a} & =\frac{I}{2 R\left(R+x_{3}\right)} \epsilon_{a b 3} x_{b}\left(1+\frac{2 R+x_{3}}{2 R^{2}\left(R+x_{3}\right)} \theta C \theta\right), \\
A_{\alpha} & =i \frac{I}{2 R^{3}}\left(\sigma_{a} C\right)_{\alpha \beta} x_{a} \theta_{\beta},
\end{aligned}
$$

with $I=1$. $A_{a}$ and $A_{\alpha}$ form a super-vector multiplet under the $O S p(1 \mid 2)$ transformation, and they would be interpreted as photon and photino fields, respectively. The field strengths are defined by

$$
F_{a b}=\partial_{a} A_{b}-\partial_{b} A_{a}, \quad F_{a \alpha}=\partial_{a} A_{\alpha}-\partial_{\alpha} A_{a}, \quad F_{\alpha \beta}=\partial_{\alpha} A_{\beta}+\partial_{\beta} A_{\alpha},
$$

and obtained as

$$
\begin{aligned}
& F_{a b}=-\frac{I}{2 R^{3}} \epsilon_{a b c} x_{c}\left(1+\frac{3}{2 R^{2}} \theta C \theta\right), \quad F_{a \alpha}=i \frac{I}{2 R^{3}}\left(\sigma_{b} C\right)_{\alpha \beta} \theta_{\beta}\left(\delta_{a b}-\frac{3}{R^{2}} x_{a} x_{b}\right), \\
& F_{\alpha \beta}=i \frac{I}{R^{3}} x_{a}\left(\sigma_{a} C\right)_{\alpha \beta}\left(1+\frac{3}{2 R^{2}} \theta C \theta\right) .
\end{aligned}
$$

Here, $I / 2$ takes integer or half-integer and denotes the quantized supermonopole charge. The magnitude of the supermonopole magnetic fields is given by

$$
B=\frac{4 \pi I / 2}{4 \pi R^{2}}=\frac{I}{2 R^{2}} .
$$

\section{The spherical SUSY quantum Hall effect}

\subsection{The spherical SUSY Landau problem [34]}

With the above set-up, we discuss one-particle problem on a supersphere in a supermonopole background. Since the particle on a supersphere is concerned, the Hamiltonian does not contain the radial part, and is simply given by the angular part

$$
H=\frac{1}{2 M R^{2}}\left(\Lambda_{a}^{2}+C_{\alpha \beta} \Lambda_{\alpha} \Lambda_{\beta}\right)
$$

where $\Lambda_{a}(a=1,2,3)$ and $\Lambda_{\alpha}(\alpha=1,2)$ represent the SUSY covariant angular momenta constructed from (2.3) with replacing the partial derivatives to the covariant derivatives:

$$
\begin{aligned}
& \Lambda_{a}=-i \epsilon_{a b c} x_{b}\left(\partial_{c}+i A_{a}\right)+\frac{1}{2} \theta_{\alpha}\left(\sigma_{a}\right)_{\alpha \beta}\left(\partial_{\beta}+i A_{\beta}\right), \\
& \Lambda_{\alpha}=\frac{1}{2}\left(C \sigma_{a}\right)_{\alpha \beta} x_{a}\left(\partial_{\beta}+i A_{\beta}\right)-\frac{1}{2} \theta_{\beta}\left(\sigma_{a}\right)_{\beta \alpha}\left(\partial_{a}+i A_{a}\right) .
\end{aligned}
$$


Here, $A_{a}$ and $A_{\alpha}$ are the supermonopole gauge fields (2.10). Since the motion of particle is confined on the supersphere, $\Lambda_{a}$ and $\Lambda_{\alpha}$ are tangent to the superface of the supersphere:

$$
\Lambda_{a} x_{a}+C_{\alpha \beta} \Lambda_{a} \theta_{\beta}=x_{a} \Lambda_{a}+C_{\alpha \beta} \theta_{\alpha} \Lambda_{\beta}=0
$$

The covariant momenta are not conserved quantities (for finite $A_{a}$ and $A_{\alpha}$ ), and they do not exactly satisfy the $O S p(1 \mid 2)$ algebra:

$$
\begin{aligned}
& {\left[\Lambda_{a}, \Lambda_{b}\right]=i \epsilon_{a b c}\left(\Lambda_{c}-\frac{I}{2 R} x_{c}\right), \quad\left[\Lambda_{a}, \Lambda_{\alpha}\right]=\frac{1}{2}\left(\sigma_{a}\right)_{\beta \alpha}\left(\Lambda_{\beta}-\frac{I}{2 R} \theta_{\beta}\right),} \\
& \left\{\Lambda_{\alpha}, \Lambda_{\beta}\right\}=\frac{1}{2}\left(C \sigma_{a}\right)_{\alpha \beta}\left(\Lambda_{a}-\frac{I}{2 R} x_{a}\right) .
\end{aligned}
$$

The "extra" terms in the right-hand-sides of (3.4) are proportional to the supermonopole magnetic fields,

$$
B_{a}=-\frac{I}{2 R^{3}} x_{a}, \quad B_{\alpha}=-\frac{I}{2 R^{3}} \theta_{\alpha} .
$$

The magnetic field $B(2.11)$ is equal to the magnitude of $B_{a}$ and $B_{\alpha}: B=\sqrt{B_{a}^{2}+C_{\alpha \beta} B_{\alpha} B_{\beta}}$. The number of the magnetic cells each of which occupies the area, $2 \pi \ell_{B}^{2}$ with magnetic length $\ell_{B}=1 / \sqrt{B}$, on the supersphere is given by

$$
N_{\Phi}=\frac{4 \pi R^{2}}{2 \pi \ell_{B}^{2}}=I
$$

Adding the angular momenta of the supermonopole fields to covariant angular momenta, the conserved $O S p(1 \mid 2)$ angular momenta are constructed as

$$
L_{a}=\Lambda_{a}-\frac{I}{2 R} x_{a}, \quad L_{\alpha}=\Lambda_{\alpha}-\frac{I}{2 R} \theta_{\alpha} .
$$

$L_{a}$ are $S U(2)$ rotation generators, and $L_{\alpha}$ play the role of supercharges in this model. It is straightforward to check that $L_{a}$ and $L_{\alpha}$ exactly satisfy the $O S p(1 \mid 2)$ algebra (2.1).

From the orthogonality relation (3.3), we obtain $L_{a}^{2}+C_{\alpha \beta} L_{\alpha} L_{\beta}=\Lambda_{a}^{2}+C_{\alpha \beta} \Lambda_{\alpha} \Lambda_{\beta}+(I / 2)^{2}$, and then the Casimir index for $L_{a}, L_{\alpha}$ is given by $J=n+I / 2(n$ corresponds to the Landau level index). Therefore, the energy eigenvalue is derived as

$$
E_{n}=\frac{1}{2 M R^{2}}\left(n\left(n+I+\frac{1}{2}\right)+\frac{I}{4}\right) .
$$

The degeneracy in $n$-th Landau level is ${ }^{4}$

$$
D_{n}=4 n+2 I+1 .
$$

In the lowest Landau level (LLL) $n=0$, the energy is

$$
E_{L L L}=\frac{I}{8 M R^{2}}=\frac{B}{4 M}
$$

\footnotetext{
${ }^{4}$ In the original system, the energy eigenvalue of $n$-th Landau level is $E_{n}=\frac{1}{2 M}\left(n(n+I+1)+\frac{I}{2}\right)$ and the degeneracy is $D_{n}=2 n+I+1$. The degeneracy in the SUSY system is almost doubly degenerate compared to the original "bosonic" system due to the existence of the "fermionic" counterpart. Especially, at $I \rightarrow \infty$ in the LLL, $D_{n=0} \rightarrow N_{\Phi}=I$ in the original model, while $D_{n=0} \rightarrow 2 N_{\Phi}=2 I$ in the SUSY model.
} 
and there are $2 I+1$ degenerate eigenstates that consist of

$$
u_{m_{1}, m_{2}}=\sqrt{\frac{I !}{m_{1} ! m_{2} !}} u^{m_{1}} v^{m_{2}}, \quad \eta_{n_{1}, n_{2}}=\sqrt{\frac{I !}{n_{1} ! n_{2} !}} u^{n_{1}} v^{n_{2}} \eta,
$$

with the constraints $m_{1}+m_{2}=I$ and $n_{1}+n_{2}=I-1 . u_{m_{1}, m_{2}}$ is a Grassmann even quantity, while $v_{n_{1}, n_{2}}$ is a Grassmann odd quantity. The eigenvalues of $L_{3}$ for $u_{m_{1}, m_{2}}$ and $\eta_{n_{1}, n_{2}}$ are explicitly given by $I, I-1, \ldots,-I+1,-I$ and $I-1 / 2, I-3 / 2, \ldots,-I+1 / 2$, respectively, and thus differ by $1 / 2$. Since $u_{m_{1}, m_{2}}$ and $v_{n_{1}, n_{2}}$ are related by the transformation generated by the fermionic operators $L_{\alpha}$, they are regarded as SUSY partners and named the supermonopole harmonics; $\eta_{n_{1}, n_{2}}$ are the "fermionic" counterpart of the original "bosonic" monopole harmonics $u_{m_{1}, m_{2}}$. The orthonormal relations are

$$
\begin{aligned}
& \int_{S^{2 \mid 2}} d \Omega_{2 \mid 2} u_{m_{1}, m_{2}}^{*} u_{m_{1}^{\prime}, m_{2}^{\prime}}=\frac{4 \pi I}{I+1} \delta_{m_{1}, m_{1}^{\prime}} \delta_{m_{2}, m_{2}^{\prime}} \\
& \int_{S^{2 \mid 2}} d \Omega_{2 \mid 2} \eta_{n_{1}, n_{2}}^{*} \eta_{n_{1}^{\prime}, n_{2}^{\prime}}=4 \pi \delta_{n_{1}, n_{1}^{\prime}} \delta_{n_{2}, n_{2}^{\prime}} \\
& \int_{S^{2 \mid 2}} d \Omega_{2 \mid 2} u_{m_{1}, m_{2}}^{*} \eta_{n_{1}, n_{2}}=0
\end{aligned}
$$

with $d \Omega_{2 \mid 2}=d \omega_{2} d \theta_{1} d \theta_{2} ; d \omega_{2}$ is the area element of two-sphere.

It should be also noted that the $u_{m_{1}, m_{2}}$ and $v_{n_{1}, n_{2}}$ are constructed by products of the components of SUSY Hopf spinor, so the $O S p(1 \mid 2)$ generators are effectively represented as

$$
L_{a}=\psi^{t} \tilde{l}_{\alpha} \frac{\partial}{\partial \psi}, \quad L_{\alpha}=\psi^{t} \tilde{l}_{\alpha} \frac{\partial}{\partial \psi}
$$

where $\tilde{l}_{a}$ and $\tilde{l}_{\alpha}$ are defined by equation (2.4). The supermonopole charge is measured by the operator:

$$
\hat{I}=u \frac{\partial}{\partial u}+v \frac{\partial}{\partial v}+\eta \frac{\partial}{\partial \eta} .
$$

Complex variables never appear in the LLL bases (3.7), and they are replaced by derivatives:

$$
\psi^{*}=\left(u^{*}, v^{*}, \eta^{*}\right)^{t} \rightarrow \frac{1}{I} \frac{\partial}{\partial \psi}=\frac{1}{I}\left(\frac{\partial}{\partial u}, \frac{\partial}{\partial v}, \frac{\partial}{\partial \eta}\right)^{t},
$$

as suggested by equations (3.8). This substitution implies that $\psi$ and $\psi^{*}$ no longer commute each other; this gives rise to NCG in LLL.

Here, we make some comments about peculiar properties of the present supersymmetry. The Hamiltonian (3.1), which is equal to the $O S p(1 \mid 2)$ Casimir operator up to constant, apparently commutes with the supercharges $L_{\alpha}$, and, in this sense, the present model possesses a supersymmetry. However, there are some differences between the present SUSY model and conventional SUSY quantum mechanics. First of all, the present model is defined on supermanifold, while basemanifolds for SUSY quantum mechanics are usually taken to be bosonic. Then, in this model, energy eigenfunctions generally depend on Grassmann odd coordinates as well as Grassmann even coordinates. (In this sense, our wavefunctions are something like superfields.) Second, the supercharges are not nilpotent: $L_{\theta_{1}}^{2}=\left(L_{x}+i L_{y}\right) / 4$ and $L_{\theta_{2}}^{2}=-\left(L_{x}-i L_{y}\right) / 4$ as suggested by the $O S p(1 \mid 2)$ algebra (2.1). Thus, the square of the supercharges acts as the ladder operators for $S U(2)$ spin 1, and the supercharges themselves are regarded as ladder operators for $S U(2)$ spin 1/2. Last, the Hamiltonian (3.1) is not given by the anticommutator of supercharges, so the lowest energy (LLL energy) is not zero but finite (3.6). Similarly, bosonic degrees of freedom do not exactly equal to fermionic ones but differ by 1 . 


\subsection{The spherical SUSY Laughlin wavefunction and excitations [35]}

Now, we are ready to discuss many-body problem. First, we construct the groundstate wavefunction of the SUSY QHE. The original Laughlin wavefunction on a two-sphere was given by

$$
\Phi=\prod_{i<j}^{N}\left(\phi_{i}^{t} C \phi_{j}\right)^{m}=\prod_{i<j}^{N}\left(u_{i} v_{j}-v_{i} u_{j}\right)^{m}
$$

where $\phi=(u, v)^{t}$ represents the original $S U(2)$ Hopf spinor and $N$ represents the total number of particles and $m$ denotes integer [29]. Thus, $\Phi$ is constructed by the product of $S U(2)$ singlets of two Hopf spinors. Then, it would be natural to adopt product of $O S p(1 \mid 2)$ singlets made of two super Hopf spinors as a SUSY Laughlin wavefunction. However, as discussed above, we cannot use complex variables to construct $O S p(1 \mid 2)$ singlet in LLL. Fortunately, the complex and the original representations are unitary equivalent, and hence it is possible to construct a singlet of two super Hopf spinors without introducing complex variables. Thus, the spherical SUSY Laughlin wavefunction is constructed as

$$
\Psi=\prod_{i<j}^{N}\left(\psi_{i}^{t} \mathcal{R} \psi_{j}\right)^{m}=\prod_{i<j}^{N}\left(u_{i} v_{j}-u_{j} v_{i}-\eta_{i} \eta_{j}\right)^{m} .
$$

Since the SUSY Laughlin wavefunction is invariant under the $O S p(1 \mid 2)$ SUSY transformation, the super partner of the SUSY Laughlin wavefunction does not exist. Acting the monopole charge operator (3.10) to the SUSY wavefunction, one may see that the monopole charge $I$ is related to $N$ and $m$ as

$$
I=m(N-1) .
$$

There may be two choices to define the filling factor $\nu$ in the SUSY QHE: $N / N_{\Phi}$ or $N / D$. In the thermodynamic limit $\left(N, I, R \rightarrow \infty\right.$ with the magnetic length $\ell_{B}$ fixed), these two definitions are different; $N / D \rightarrow N / 2 I=N / 2 N_{\Phi}$, unlike the original $\mathrm{QHE}^{5}$. It is convenient to use the definition

$$
\nu=\frac{N}{N_{\Phi}}=\frac{N}{I}
$$

The filling factor for the SUSY Laughlin wavefunction reads as $N / m(N-1)$, which tends to $\nu=1 / m$ in the thermodynamic limit.

It is also possible to construct a pseudo-potential Hamiltonian whose zero-energy eigenstate is the SUSY Laughlin wavefunction. The SUSY Laughlin wavefunction is $O S p(1 \mid 2)$ symmetric, and does not have any components whose 3rd component of the two-body angular momentum eigenvalue is greater than $m(N-2)=I-m$. Then, the SUSY Laughlin wavefunction does not contain any components whose two-body $O S p(1 \mid 2)$ Casimir index $J$ is greater than $I-m$. From this observation, the pseudo-potential Hamiltonian is derived as

$$
\hat{V}=\sum_{J=I-m+1 / 2, I-m+1, \ldots, I} V_{J} \cdot P_{J}\left(L_{a}(i) L_{a}(j)+C_{\alpha \beta} L_{\alpha}(i) L_{\beta}(j)\right)
$$

\footnotetext{
${ }^{5}$ In the original QHE, these two definitions coincide in the thermodynamic limit: $N / D \rightarrow N / I=N / N_{\Phi}$.
} 
where coefficients $V_{J}$ are taken to be positive, and $P_{J}$ is given by

$$
\begin{aligned}
P_{J}( & \left.L_{a}(i) L_{a}(j)+C_{\alpha \beta} L_{\alpha}(i) L_{\beta}(j)\right) \\
& =\prod_{J^{\prime} \neq J} \frac{\left(L_{a}(i)+L_{a}(j)\right)^{2}+C_{\alpha \beta}\left(L_{\alpha}(i)+L_{\alpha}(j)\right)\left(L_{\beta}(i)+L_{\beta}(j)\right)-J^{\prime}\left(J^{\prime}+\frac{1}{2}\right)}{J\left(J+\frac{1}{2}\right)-J^{\prime}\left(J^{\prime}+\frac{1}{2}\right)} \\
& =\prod_{J^{\prime} \neq J} \frac{2 L_{a}(i) L_{a}(j)+2 C_{\alpha \beta} L_{\alpha}(i) L_{\beta}(j)+\frac{I}{2}(I+1)-J^{\prime}\left(J^{\prime}+\frac{1}{2}\right)}{J\left(J+\frac{1}{2}\right)-J^{\prime}\left(J^{\prime}+\frac{1}{2}\right)} .
\end{aligned}
$$

$P_{J}$ denotes the projection operator to the subspace of two-body $O S p(1 \mid 2)$ Casimir index $J$. With positive coefficients $V_{J}$, the lowest energy eigenvalue of the Hamiltonian (3.15) is zero, and the pseudo-potential Hamiltonian does not have any component $J<I-m$. Therefore, the SUSY Laughlin wavefunction is the zero-energy exact groundstate of the Hamiltonian ${ }^{6}$.

Quasi-hole (= vortex) and quasi-particle (= anti-vortex) operators are respectively constructed as

$$
\begin{aligned}
& A(\chi)^{\ddagger}=\prod_{i} \psi_{i} \mathcal{R} \chi=\prod_{i}\left(b v_{i}-a u_{i}-\xi \eta_{i}\right), \\
& A(\chi)=\prod_{i} \chi^{\ddagger} \mathcal{R}^{t} \frac{\partial}{\partial \psi_{i}}=\prod_{i}\left(b^{*} \frac{\partial}{\partial v_{i}}-a^{*} \frac{\partial}{\partial u_{i}}-\xi^{*} \frac{\partial}{\partial \eta_{i}}\right),
\end{aligned}
$$

where $\chi \equiv(a, b, \xi)^{t}$ is a normalized constant spinor, which specifies the position at which the quasi-hole (quasi-particle) is created on the supersphere by relations: $\Omega_{a}=2 \chi^{\ddagger} l_{a} \chi$ and $\Omega_{\alpha}=2 \chi^{\ddagger} l_{\alpha} \chi$. Their commutation relations are

$$
\left[A(\chi), A(\chi)^{\ddagger}\right]=1, \quad\left[A(\chi), A\left(\chi^{\prime}\right)\right]=\left[A^{\ddagger}(\chi), A^{\ddagger}\left(\chi^{\prime}\right)\right]=0 .
$$

Similarly, the commutation relation between the quasi-hole operator and the $O S p(1 \mid 2)$ operators in the direction $\left(\Omega_{a}, \Omega_{\alpha}\right)$ is given by

$$
\left[\Omega_{a}(\chi) L_{a}+C_{\alpha \beta} \Omega_{\alpha}(\chi) L_{\beta}, A(\chi)\right]=\frac{N}{2} A(\chi) .
$$

This implies that the creation of quasi-hole increases the angular momentum in the direction of the point $\left(\Omega_{a}, \Omega_{\alpha}\right)$ by $N / 2$. Physically, it is understood as follows. The creation of quasi-hole pushes the particles on the SUSY Laughlin state downward from the point $\left(\Omega_{a}, \Omega_{\alpha}\right)$, so the charge deficit which we identify quasi-particle is generated at the point. The relation (3.14) suggests that the excess of unit magnetic flux, $\delta I=1$, induces excitation with fractional charge $e^{*}=1 / m$. Thus, the quasi-particle excitation in SUSY QHE at $\nu=1 / m$ carries the fractional charge $1 / m$ as in the original QHE [29], and the fractional charge is induced by bosonic and fermionic Hall currents as suggested by equations (4.4).

\section{Emergence of non-anti-commutative geometry [34, 35]}

Originally, $x_{a}$ and $\theta_{\alpha}$ were the classical coordinates on supersphere and not operators, while, in the LLL, they are effectively regarded as operators. It is because, in the LLL limit $(M \rightarrow 0)$ the covariant angular momenta can be neglected (see equations (6.2)), and $x_{a}$ and $\theta_{\alpha}$ are reduced to the $O S p(1 \mid 2)$ operators as indicated by equation (3.5):

$$
\left(x_{a}, \theta_{\alpha}\right) \rightarrow\left(X_{a}, \Theta_{\alpha}\right) \equiv-\alpha\left(L_{a}, L_{\alpha}\right),
$$

\footnotetext{
${ }^{6}$ It is reported that in scalar field theories on supersphere effective potentials are not generally bounded below, so the groundstates are not stable [20]. However, such problem cannot be applied to SUSY QHE, since the pseudo-potential Hamiltonian (3.15) has the lowest eigenvalue and the energies are bounded.
} 
with $\alpha=2 R / I$. Thus, in the LLL, $x_{a}$ and $\theta_{\alpha}$ become operators that satisfy the SUSY NC algebra:

$$
\begin{aligned}
& {\left[X_{a}, X_{b}\right]=-i \alpha \epsilon_{a b c} X_{c}, \quad\left[X_{a}, \Theta_{\alpha}\right]=-\frac{\alpha}{2}\left(\sigma_{a}\right)_{\beta \alpha} \Theta_{\beta},} \\
& \left\{\Theta_{\alpha}, \Theta_{\beta}\right\}=-\frac{\alpha}{2}\left(C \sigma_{a}\right)_{\alpha \beta} X_{a} .
\end{aligned}
$$

The first relation manifests the noncommutativity in the LLL, and the second relation suggests the non-trivial "coupling" between the bosonic and fermionic operators. The latter relation reflects the non-anti-commutative geometry in the SUSY LLL. The fuzzy super manifold introduced by the algebraic relation (4.2) is known as the fuzzy supersphere [36, 37] $]^{7}$. Thus, SUSY NCG are nicely realized in the formulation of the SUSY QHE. Alternatively, one may find the emergence of fuzzy supersphere by the following derivation. Complex variables are regarded as derivatives in the LLL (3.11), and the Hopf map (2.6) is reduced to

$$
X_{a}=-\alpha \psi^{t} \tilde{l_{a}} \frac{\partial}{\partial \psi}, \quad \Theta_{\alpha}=-\alpha \psi^{t} \tilde{l_{\alpha}} \frac{\partial}{\partial \psi} .
$$

Apparently, $X_{a}$ and $\Theta_{\alpha}$ satisfy the algebra of fuzzy supersphere. By comparison of equation (4.3) and equation (3.9), the equivalence between $\left(L_{a}, L_{\alpha}\right)$ and $\left(X_{a}, \Theta_{\alpha}\right)$ in LLL (4.1) is also confirmed. Equations (4.2) imply that the super Hall currents $I_{a}=\frac{d}{d t} X_{a}, I_{\alpha}=\frac{d}{d t} \Theta_{\alpha}$ satisfy the relations,

$$
\begin{aligned}
I_{a} & =-i\left[X_{a}, V\right]=(\alpha R)^{2} \epsilon_{a b c} B_{b} E_{c}-i \frac{1}{2}(\alpha R)^{2}\left(\sigma_{a} C\right)_{\alpha \beta} B_{\alpha} E_{\beta}, \\
I_{\alpha} & =-i\left[\Theta_{\alpha}, V\right]=i \frac{1}{2}(\alpha R)^{2}\left(\sigma_{a}\right)_{\beta \alpha} B_{a} E_{\beta}+i \frac{1}{2}(\alpha R)^{2}\left(\sigma_{a}\right)_{\beta \alpha} B_{\beta} E_{a},
\end{aligned}
$$

where $E_{a}=-\partial_{a} V, E_{\alpha}=C_{\alpha \beta} \partial_{\beta} V$. With equations (4.4), it is checked that the super Hall currents are orthogonal to the super electric fields and the super magnetic fields, respectively:

$$
E_{a} I_{a}+C_{\alpha \beta} E_{\alpha} I_{\beta}=0, \quad B_{a} I_{a}+C_{\alpha \beta} B_{\alpha} I_{\beta}=0 .
$$

Around the north-pole of the fuzzy supersphere $X_{3} \approx \alpha I / 2$, the SUSY NC algebra (4.2) is reduced to that on the NC superplane,

$$
\left[X_{i}, X_{j}\right]=i \epsilon_{i j} \ell_{B}^{2}, \quad\left[X_{i}, \Theta_{\alpha}\right]=0, \quad\left\{\Theta_{\alpha}, \Theta_{\beta}\right\}=\left(\sigma_{1}\right)_{\alpha \beta} \ell_{B}^{2} .
$$

Such planar reductions were well examined by the Inönü-Wigner contraction technique in more general contexts [39]. As we shall see below, the planar SUSY QHE naturally manifests the planar SUSY NC algebra in LLL.

\section{The planar SUSY quantum Hall effect}

\subsection{The generators on the superplane and stereographic projection $[40,41]$}

Using the Inönü-Wigner contraction, we derive the symmetry generators on the superplane from the $O S p(1 \mid 2)$ generators. We apply a symmetric scaling to the $O S p(1 \mid 2)$ generators as

$$
L_{i} \rightarrow \epsilon T_{i}, \quad L_{\alpha} \rightarrow \epsilon T_{\alpha}, \quad L_{3} \rightarrow L_{\perp} .
$$

By taking the limit $\epsilon \rightarrow 0$, the $O S p(1 \mid 2)$ SUSY commutation relations are reduced to the translation and rotation algebras on the superplane,

$$
\begin{aligned}
& {\left[T_{i}, T_{j}\right]=0, \quad\left[T_{i}, L_{\perp}\right]=-i \epsilon_{i j} T_{j},} \\
& {\left[T_{i}, T_{\alpha}\right]=0,} \\
& \left\{T_{\alpha}, T_{\beta}\right\}=0, \quad\left[T_{\alpha}, L_{\perp}\right]= \pm \frac{1}{2} T_{\alpha},
\end{aligned}
$$

\footnotetext{
${ }^{7}$ The fuzzy supersphere is a classical solution of supermatrix model [16]. A nice review of mathematics and physical applications of fuzzy sphere and fuzzy supersphere is found in [38].
} 
where, in the latter equation, + and - correspond to $\alpha=1$ and $\alpha=2$, respectively. The differential operators that satisfy (5.1) denote the translation generators and the perpendicular angular momentum on the superplane. They are explicitly represented as

$$
T_{i}=-i \partial_{i}, \quad T_{\alpha}=-i \partial_{\alpha}, \quad L_{\perp}=z \frac{\partial}{\partial z}-z^{*} \frac{\partial}{\partial z^{*}}+\frac{1}{2} \theta \frac{\partial}{\partial \theta}-\frac{1}{2} \theta^{*} \frac{\partial}{\partial \theta^{*}} .
$$

The first two terms in $L_{\perp}$ denote the conventional orbital angular momentum and count the difference between the powers of $z$ and $z^{*}$. Essentially, $z$ corresponds to the right-handed orbital rotation, and $z^{*}$ the left-handed orbital rotation. Similarly, $\theta$ may be regarded as the right-handed spin rotation and $\theta^{*}$ the left-handed spin rotation. Indeed, the factor $1 / 2$ in front of the last two terms in $L_{\perp}$ implies $\theta$ and $\theta^{*}$ carry the spin-up and spin-down degree of freedom, respectively.

Introducing the super stereographic coordinates $(z, \theta)$

$$
z \equiv \frac{v}{u}=\frac{x_{1}+i x_{2}}{R+x_{3}}\left(1+\frac{1}{2 R\left(R+x_{3}\right)} \theta C \theta\right), \quad \theta \equiv \frac{\eta}{u}=\theta_{1}+z \theta_{2}
$$

we simply express the SUSY Hopf spinor as

$$
\psi=\frac{1}{\sqrt{1+z z^{*}+\theta \theta^{*}}}\left(\begin{array}{l}
1 \\
z \\
\theta
\end{array}\right) .
$$

The supermonopole harmonics are also rewritten as

$$
u_{m_{1}, m_{2}}=\sqrt{\frac{I !}{m_{1} ! m_{2} !}} z^{m_{2}}\left(\frac{1}{1+z z^{*}+\theta \theta^{*}}\right)^{\frac{I}{2}}, \quad \eta_{n_{1}, n_{2}}=\sqrt{\frac{I !}{n_{1} ! n_{2} !}} z^{n_{2}} \theta\left(\frac{1}{1+z z^{*}+\theta \theta^{*}}\right)^{\frac{I}{2}}
$$

and, in the thermodynamic limit, they become

$$
\phi_{m}=\sqrt{\frac{2^{m+1}}{\pi m !}} z^{m} e^{-z z^{*}-\theta \theta^{*}}, \quad \psi_{m-\frac{1}{2}}=\sqrt{\frac{2^{m}}{\pi(m-1) !}} z^{m-1} \theta e^{-z z^{*}-\theta \theta^{*}} .
$$

Their coefficients are chosen to satisfy the orthonormal conditions:

$$
\begin{aligned}
& \int d z d z^{*} d \theta d \theta^{*} \phi_{m}^{*} \phi_{m}^{\prime}=\int d z d z^{*} d \theta d \theta^{*} \psi_{m-1 / 2}^{*} \psi_{m^{\prime}-1 / 2}=\delta_{m m^{\prime}}, \\
& \int d z d z^{*} d \theta d \theta^{*} \phi_{m}^{*} \psi_{m^{\prime}-1 / 2}=0 .
\end{aligned}
$$

As found in equations (5.3), the complex variables $z^{*}$ and $\theta^{*}$ do not appear in the LLL up to the exponential. In the LLL, $z^{*}$ and $\theta^{*}$ are equivalent to derivatives:

$$
\left(z^{*}, \theta^{*}\right) \rightarrow\left(-\frac{\partial}{\partial z},-\frac{\partial}{\partial \theta}\right) .
$$

It is apparent that operations of the derivatives to (5.3) are same as of the complex variables.

\subsection{The planar SUSY Landau problem [40]}

As the planar SUSY Hamiltonian we adopt the following operator

$$
H=-\frac{1}{2 M}\left(D_{i}^{2}+C_{\alpha \beta} D_{\alpha} D_{\beta}\right),
$$


where $D_{i}$ and $D_{\alpha}$ denote the SUSY covariant derivatives defined by $D_{i}=\partial_{i}-i A_{i}$ and $D_{\alpha}=$ $\partial_{\alpha}-i A_{\alpha}$, with $A_{i}=B / 2 \epsilon_{i j} x_{j}$ and $A_{\alpha}=B / 2\left(\sigma_{1}\right)_{\alpha \beta} \theta_{\beta}$. Identifying the stereographic coordinates (5.2) with $x_{i}$ and $\theta_{\alpha}$ :

$$
z=\frac{1}{2 \ell_{B}}(x+i y), \quad z^{*}=\frac{1}{2 \ell_{B}}(x-i y), \quad \theta=\frac{1}{\sqrt{2} \ell_{B}} \theta_{1}, \quad \theta^{*}=\frac{1}{\sqrt{2} \ell_{B}} \theta_{2},
$$

it is easily shown that LLL bases (5.3) are zero-energy degenerate groundstates of the SUSY Hamiltonian.

The SUSY covariant derivatives $D_{i}$ and $D_{\alpha}$ satisfy the algebra,

$$
\left[D_{i}, D_{j}\right]=i B \epsilon_{i j}, \quad\left\{D_{\alpha}, D_{\beta}\right\}=-B\left(\sigma_{1}\right)_{\alpha \beta}, \quad\left[D_{i}, D_{\alpha}\right]=0 .
$$

The SUSY center-of-mass coordinates are constructed as

$$
X_{i}=x_{i}+i \ell_{B}^{2} D_{j}, \quad \Theta_{\alpha}=\theta_{\alpha}+\ell_{B}^{2} D_{\alpha},
$$

and satisfy the SUSY NC relations,

$$
\left[X_{i}, X_{j}\right]=i \ell_{B}^{2} \epsilon_{i j}, \quad\left\{\Theta_{\alpha}, \Theta_{\beta}\right\}=\ell_{B}^{2}\left(\sigma_{1}\right)_{\alpha \beta}, \quad\left[X_{i}, \Theta_{\alpha}\right]=0 .
$$

In the LLL $\operatorname{limit}^{8}(M \rightarrow 0), x_{i}$ and $\theta_{\alpha}$ are reduced to $X_{i}$ and $\Theta_{\alpha}$ respectively, so the SUSY NC relations (5.9) are realized in the planar SUSY QHE as expected. Equations (5.6) and equations (5.9) suggest that $z$ and $z^{*}$ are no longer commutative but noncommutative, and similarly $\theta$ and $\theta^{*}$ are no longer anti-commutative but non-anti-commutative in the LLL. This observation is consistent with the substitution (5.4). From two-sets of SUSY commutation relations (5.7)-(5.9), two sets of bosonic and fermionic raising and lowering operators are naturally defined:

$$
a=-i \frac{\ell_{B}}{\sqrt{2}}\left(D_{x}+i D_{y}\right), \quad a^{\dagger}=-i \frac{\ell_{B}}{\sqrt{2}}\left(D_{x}-i D_{y}\right), \quad \alpha=i \ell_{B} D_{\theta_{2}}, \quad \alpha^{\dagger}=i \ell_{B} D_{\theta_{1}},
$$

and

$$
b=\frac{1}{\sqrt{2} \ell_{B}}(X-i Y), \quad b^{\dagger}=\frac{1}{\sqrt{2} \ell_{B}}(X+i Y), \quad \beta=\frac{1}{\ell_{B}} \Theta_{2}, \quad \beta^{\dagger}=\frac{1}{\ell_{B}} \Theta_{1} .
$$

With such SUSY raising and lowering operators, one may construct two kinds of supercharges:

$$
\left(Q, Q^{\dagger}\right)=\left(a^{\dagger} \alpha, \alpha^{\dagger} a\right), \quad\left(\tilde{Q}, \tilde{Q}^{\dagger}\right)=\left(b^{\dagger} \beta, \beta^{\dagger} b\right) .
$$

The first set and the second set are anti-commutative each other, and these sets of supercharges generate two independent SUSY transformations that we call $Q$-SUSY and $\tilde{Q}$-SUSY. With use of $Q$-SUSY generators, the Hamiltonian (5.5) is rewritten as

$$
H=\omega\left\{Q, Q^{\dagger}\right\}=\omega\left(a^{\dagger} a+\alpha^{\dagger} \alpha\right) .
$$

Its eigenenergy is

$$
E_{n}=\omega n,
$$

where $n$ takes integer that specifies the SUSY Landau level. Since the Hamiltonian commutes with $\tilde{Q}$ and $\tilde{Q}^{\dagger}$ in addition to $Q$ and $Q^{\dagger}$, the planar SUSY model possesses $\mathcal{N}=2$ SUSY in total. The $\mathcal{N}=2$ SUSY multiplets are constructed by acting the operators

$$
\begin{aligned}
& \frac{1}{\sqrt{n ! m !}} a^{\dagger^{n}} b^{\dagger^{m}}, \quad \frac{1}{\sqrt{n !(m-1) !}} a^{\dagger^{n}} \beta^{\dagger} b^{\dagger^{m-1}}, \quad \frac{1}{\sqrt{(n-1) ! m !}} \alpha^{\dagger} a^{\dagger^{n-1}} b^{\dagger^{m}}, \\
& \frac{1}{\sqrt{(n-1) !(m-1) !}} \alpha^{\dagger} a^{\dagger^{n-1}} \beta^{\dagger} b^{\dagger^{m-1}}
\end{aligned}
$$

\footnotetext{
${ }^{8}$ The LLL limit is formally realized by neglecting the SUSY covariant derivatives $D_{i}$ and $D_{\alpha}$.
} 


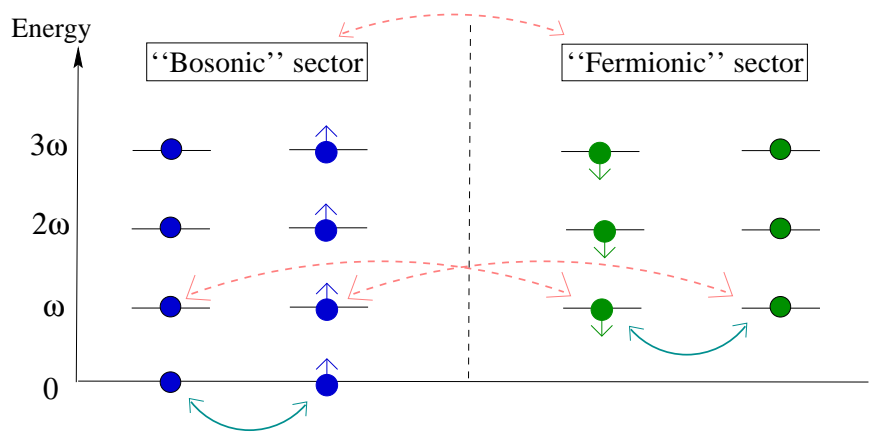

Figure 1. The "balls" correspond to the states given by equation (5.10). The solid curved arrows represent the $Q$-SUSY transformations, while the dotted curved arrows represent the $\tilde{Q}$-SUSY transformations.

to the vacuum (see Fig. 1). Specifically, the LLL $(n=0)$-sector consists of

$$
\left|\phi_{m}\right\rangle=\frac{1}{\sqrt{n ! m !}} a^{\dagger^{n}} b^{\dagger^{m}}|0\rangle, \quad\left|\psi_{m-\frac{1}{2}}\right\rangle=\frac{1}{\sqrt{n !(m-1) !}} a^{\dagger^{n}} \beta^{\dagger} b^{\dagger^{m-1}}|0\rangle .
$$

These are supermultiplet related by $\tilde{Q}$-SUSY. Thus, while the LLL is the "vacuum" of the $Q$ SUSY, there still exist $\mathcal{N}=1$ SUSY degeneracies due to $\tilde{Q}$-SUSY. The LLL wavefunctions (5.3) are reproduced from equations (5.11) with the vacuum $\phi_{0}=\frac{1}{\sqrt{\pi}} e^{-z z^{*}-\theta \theta^{*}}$.

The perpendicular angular momentum $L_{\perp}$ is expressed by the SUSY creation and annihilation operators as

$$
L_{\perp}=\left(b^{\dagger} b+\frac{1}{2} \beta^{\dagger} \beta\right)-\left(a^{\dagger} a+\frac{1}{2} \alpha^{\dagger} \alpha\right)
$$

and the commutation relations between the supercharges and $L_{\perp}$ are

$$
\left[L_{\perp}, Q\right]=-\frac{1}{2} Q, \quad\left[L_{\perp}, Q^{\dagger}\right]=\frac{1}{2} Q^{\dagger}, \quad\left[L_{\perp}, \tilde{Q}\right]=\frac{1}{2} \tilde{Q}, \quad\left[L_{\perp}, \tilde{Q}^{\dagger}\right]=-\frac{1}{2} \tilde{Q}^{\dagger} .
$$

Thus, the supercharges are spin $1 / 2$ operators. The magnitudes of the perpendicular angular momenta for the $\mathcal{N}=2$ SUSY multiplets $(5.10)$ are respectively given by $(m-n),\left(m-n-\frac{1}{2}\right)$,

$\left(m-n+\frac{1}{2}\right)$ and $(m-n)$. The multiplets possess the same orbital angular momentum: $(m-n)$, while their spins are different: $0,1 / 2,0$ and $-1 / 2$ (Fig. 1 ).

\subsection{The SUSY Laughlin wavefunction [41]}

In the thermodynamic limit, the spherical SUSY Laughlin wavefunction (3.13) is transformed to the planar SUSY Laughlin wavefunction:

$$
\Psi=\prod_{i<j}^{N}\left(z_{i}-z_{j}+\theta_{i} \theta_{j}\right)^{m} e^{-\sum_{i}\left(z_{i} z_{i}^{*}+\theta_{i} \theta_{i}^{*}\right)},
$$

where $z$ and $\theta$ are the stereographic coordinates. To explore its physical meaning, it is important to notice that the SUSY Laughlin wavefunction is rewritten in the form:

$$
\begin{aligned}
\Psi= & \exp \left(m \sum_{i<j} \frac{\theta_{i} \theta_{j}}{z_{i}-z_{j}}\right) \cdot \Phi=\Phi+m \sum_{i<j} \frac{\theta_{i} \theta_{j}}{z_{i}-z_{j}} \Phi \\
& +\frac{m^{2}}{2}\left(\sum_{i<j} \frac{\theta_{i} \theta_{j}}{z_{i}-z_{j}}\right)^{2} \Phi+\cdots+\frac{m^{\frac{N}{2}}}{(N / 2) !} \theta_{1} \theta_{2} \cdots \theta_{N} \cdot \operatorname{Pf}\left(\frac{1}{z_{i}-z_{j}}\right) \Phi,
\end{aligned}
$$




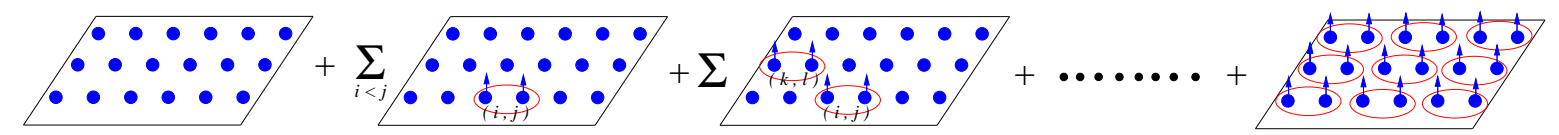

Figure 2. The graphical representation for the expansion (5.12). Each circle represents the $p$-wave pairing on the Laughlin state. In the $n$-th component wavefunction of the expansion, the pairing operator acts to the Laughlin state $n-1$ times and constitute $n-1$ spin-polarized $p$-wave pairings.

where $\Phi$ denotes the planar version of the original Laughlin wavefunction (3.12),

$$
\Phi=\prod_{i<j}\left(z_{i}-z_{j}\right)^{m} e^{-\sum_{i}\left(z_{i} z_{i}^{*}+\theta_{i} \theta_{i}^{*}\right)} .
$$

In the second equation of equation (5.12), we expanded the exponential in terms of Grassmann quantity, $\sum_{i<j} \theta_{i} \theta_{j} /\left(z_{i}-z_{j}\right)$, which we call the pairing operator hereafter. Since $\theta$ carries spin $1 / 2$ degree of freedom, the numerator $\theta_{i} \theta_{j}$ acts to attach spin $1 / 2$ to each of the original Laughlin spinless particles $i$ and $j$. Meanwhile, the denominator $1 /\left(z_{i}-z_{j}\right)$ is a solution of the $2 \mathrm{D}$ Schrödinger equation with attractive contact interaction, and represents a $p$-wave pairing state of $i$ and $j$ particles. Then, in total, the pairing operator $\theta_{i} \theta_{j} /\left(z_{i}-z_{j}\right)$ may be regarded as an operator that forms a spin-polarized $p$-wave pairing state of $i, j$ particles on the Laughlin state. With this interpretation, the expansion (5.12) now has the following physical meaning. Apparently, the 1st component of the expansion is the original Laughlin wavefunction. In the 2nd component, the pairing operator acts to the original Laughlin wavefunction once, and one $p$-wave pairing state is generated on the Laughlin state. Similarly, in the 3rd component, the pairing operator acts on the Laughlin function twice, and two $p$-wave pairings are generated on the Laughlin state. Repeating this procedure, we finally arrive at the state where all particles form $p$-wave pairings with polarized spins (Fig. 2). This state is nothing but Moore-Read state [42] that was proposed as a candidate groundstate at even denominator fillings [43] ${ }^{9}$. Indeed, Pfaffian form proposed by Moore and Read appears as the last component wavefunction in the expansion (5.12). Thus, rather unexpectedly, the SUSY provides a unified formulation of Laughlin and Moore-Read states.

\section{SUSY Chern-Simons effective field theory}

In this section, we explore a field theoretical description for the SUSY quantum Hall effect. First, we provide one-particle Lagrange formalism which is complementary to the Hamilton formalism developed above. Next, we construct a Chern-Simons field theory for the present SUSY many-body problem.

\subsection{One-particle Lagrange formalism [46]}

The one-particle Lagrangian subject to the surface of the supersphere is given by

$$
L=\frac{M}{2}\left(\dot{x}_{a}^{2}+C_{\alpha \beta} \dot{\theta}_{\alpha} \dot{\theta}_{\beta}\right)+\dot{x}_{a} A_{a}+\dot{\theta}_{\alpha} A_{\alpha}-V,
$$

\footnotetext{
${ }^{9}$ Especially, the Moore-Read state is a most promising candidate for the QH groundstate at the filling 5/2, where $p$-wave pairing bosons condense to form a "bosonic" $\mathrm{QH}$ liquid.
} 
where $x_{a}$ and $\theta_{\alpha}$ satisfy the constraint (2.7). The covariant angular momenta corresponding to $(3.2)$ are

$$
\begin{aligned}
& \Lambda_{a}=M \epsilon_{a b c} x_{b} \dot{x}_{c}+i \frac{M}{2} \theta_{\alpha}\left(\sigma_{a} C\right)_{\alpha \beta} \dot{\theta}_{\beta}, \\
& \Lambda_{\alpha}=i \frac{M}{2} x_{a}\left(\sigma_{a}\right)_{\beta \alpha} \dot{\theta}_{\beta}-i \frac{M}{4} \theta_{\beta}\left(\sigma_{a}\right)_{\beta \alpha} \dot{x}_{a} .
\end{aligned}
$$

It is straightforward to confirm the orthogonality (3.3) with this expression. By introducing the Lagrange multiplier $\lambda$, the equations of motion are derived as

$$
\begin{aligned}
& M \ddot{x}_{a}=F_{a b} \dot{x}_{b}-F_{a \alpha} \dot{\theta}_{\alpha}+E_{a}+\lambda x_{a}, \\
& M \ddot{\theta}_{\alpha}=C_{\alpha \beta}\left(F_{a \beta} \dot{x}_{a}+F_{\beta \gamma} \dot{\theta}_{\gamma}\right)+E_{\alpha}+\lambda \theta_{\alpha} .
\end{aligned}
$$

From these, the Lagrangian multiplier is obtained as

$$
\lambda=-M\left(\dot{x}_{a}^{2}+C_{\alpha \beta} \dot{\theta}_{\alpha} \dot{\theta}_{\beta}\right)-\left(E_{a} x_{a}+C_{\alpha \beta} E_{\alpha} \theta_{\beta}\right) .
$$

Equations (6.3) suggest that the super drift motion of the particle:

$$
E_{a} \dot{x}_{a}+C_{\alpha \beta} E_{\alpha} \dot{\theta}_{\beta}=M\left(\dot{x}_{a} \ddot{x}_{a}+C_{\alpha \beta} \dot{\theta}_{\alpha} \ddot{\theta}_{\beta}\right) .
$$

In the LLL limit $(M \rightarrow 0)$, the right-hand-side of (6.4) becomes zero and the electric fields are orthogonal to the SUSY Hall currents as previously discussed (4.5). When the electric fields are turned off, the velocity and the acceleration becomes orthogonal; this represents the circular motion around the center-of-mass coordinates. In the LLL, the one-particle Lagrangian (6.1) is reduced to

$$
L_{L L L}=\dot{x}_{a} A_{a}+\dot{\theta}_{\alpha} A_{\alpha}-V .
$$

Since the variation of the SUSY Hopf spinor provides the gauge fields (2.9), the gauge interaction term is simply represented as

$$
\dot{x}_{a} A_{a}+\dot{\theta}_{\alpha} A_{\alpha}=-i I \psi^{\ddagger} \frac{d}{d t} \psi .
$$

It is quite simple to see the realization of the SUSY NCG with use of the LLL Lagrangian. Regarding the Hopf spinor as fundamental variables, the canonical momentum to $\psi$ is given by

$$
\pi=\partial L_{L L L} / \partial \dot{\psi}=-i I \psi^{\ddagger} .
$$

The canonical quantization condition between $\psi$ and $\pi$ induces the relation:

$$
\left[\psi, \psi^{\ddagger}\right]_{ \pm}=-\frac{1}{I},
$$

where + denotes the commutator used for Grassmann even-even, even-odd and odd-even components of $\psi$ and $\psi^{\ddagger}$, while - denotes the anticommutator used for Grassmann odd-odd case. Thus, we reproduce the results of equation (3.11); complex variables are equivalent to derivatives in LLL.

In the planar limit $x_{3} \approx R$, the one-particle Lagrangian is reduced to

$$
L=\frac{M}{2}\left(\dot{x}_{i}^{2}+C_{\alpha \beta} \dot{\theta}_{\alpha} \dot{\theta}_{\beta}\right)-\frac{B}{2} \epsilon_{i j} \dot{x}_{i} x_{j}-i \frac{B}{2}\left(\sigma_{1}\right)_{\alpha \beta} \dot{\theta}_{\alpha} \theta_{\beta} .
$$


The canonical momenta $p_{i}=\frac{\partial}{\partial \dot{x}_{i}} L, p_{\alpha}=\frac{\partial}{\partial \dot{\theta}_{\alpha}} L$ are calculated as

$$
p_{i}=M \dot{x}_{i}-\frac{B}{2} \epsilon_{i j} x_{j}, \quad p_{\alpha}=M C_{\alpha \beta} \dot{\theta}_{\beta}-i \frac{B}{2}\left(\sigma_{1}\right)_{\alpha \beta} \theta_{\beta},
$$

and the Hamiltonian is obtained as

$$
H=\frac{1}{2 M}\left(p_{i}+\frac{B}{2} \epsilon_{i j} x_{j}\right)^{2}+\frac{1}{2 M} C_{\alpha \beta}\left(p_{\alpha}+i \frac{B}{2}\left(\sigma_{1} \theta\right)_{\alpha}\right)\left(p_{\beta}+i \frac{B}{2}\left(\sigma_{1} \theta\right)_{\beta}\right) .
$$

Imposing the canonical quantization conditions, $\left[x_{i}, p_{j}\right]=i \delta_{i j}$ and $\left\{\theta_{\alpha}, p_{\beta}\right\}=i \delta_{\alpha \beta}$, it is straightforward to derive the quantum mechanical Hamiltonian (5.5). The relation (6.5) suggests that, in the LLL limit, the momenta are reduced to the coordinates; $p_{i} \rightarrow-B / 2 \epsilon_{i j} x_{j}$ and $p_{\alpha} \rightarrow-i B / 2\left(\sigma_{1}\right)_{\alpha \beta} \theta_{\beta}$, and then $x_{i}$ and $\theta_{\alpha}$ satisfy the SUSY noncommutative algebra (4.6).

\subsection{Charge-flux duality [45]}

It is well known that the Chern-Simons field theory nicely describes the low energy dynamics of QHE [44]. Since the Chern-Simons coupling induces the statistical transformation specific to 3D space-time, the Chern-Simons theory plays a crucial role for the field theoretical description of anyons in QHE. In 3D particle-magnetic flux system, there is another important concept known as the charge-flux duality. The charge-flux duality is referred to the interchangeability of the matter current $J_{a}$ and the field strength $F_{a b}(a, b=1,2,3)$. (Here, Wick-rotated 3D space-time $\mathbb{R}^{3}$ is considered.) Thanks to the existence of the 3 -rank antisymmetric tensor, $\epsilon_{a b c}$, in $3 \mathrm{D}$, 2-rank antisymmetric tensor is transferred to vector:

$$
F_{a} \equiv \frac{1}{2} \epsilon_{a b c} F_{b c},
$$

and hence there is one-to-one correspondence between $J_{a}$ and $F_{a b}$. The charge conservation law $\partial_{a} J_{a}=0$ is also consistently transfered to the Bianchi identity $\partial_{a} F_{a}=0$ in the dual picture. The CS theory also provides an appropriate field theoretical framework to realize the charge-flux duality. The CS Lagrangian coupled to the matter current is given by

$$
\mathcal{L}_{C S}=A_{a} J_{a}+\frac{1}{4 m \pi} A_{a} F_{a},
$$

where $1 / m$ represents the CS coupling (that corresponds to the filling factor in QHE). The equation of motion for $A_{3}$ is

$$
m \rho=\rho_{\Phi},
$$

where $\rho$ represents the particle density $J_{3}$, and $\rho_{\Phi}$ represents the CS magnetic flux density $B / 2 \pi$. This relation manifests that the $m$-CS fluxes are attached to each particle. Since the currents in the original system correspond to dual field strengths, the Lagrangian (6.6) may be rewritten as

$$
\mathcal{L}_{C S}=A_{a} \tilde{F}_{a}+\frac{1}{4 m \pi} A_{a} F_{a}
$$

where $\tilde{F}_{a}$ denote the dual field strengths. Integrating out the original CS fields, we obtain the dual Lagrangian expressed by the dual CS fields,

$$
\tilde{\mathcal{L}}_{C S}=-m \pi \tilde{A}_{a} \tilde{F}_{a} .
$$

The CS coupling in the dual CS Lagrangian is inverse to that in the original CS Lagrangian; the strong CS coupling region in the original system corresponds to the weak coupling region in the dual system, and vice versa ${ }^{10}$ (see Fig. 3 also). The charge-flux duality is a very important concept for the study of topological objects, since the existence of the duality permits us to switch to the dual description where topological objects arise as fundamental excitations.

\footnotetext{
${ }^{10}$ In this sense, the charge-flux duality corresponds to the $S$-dual transformation of the Chern-Simons coupling in the modern string theory language.
} 


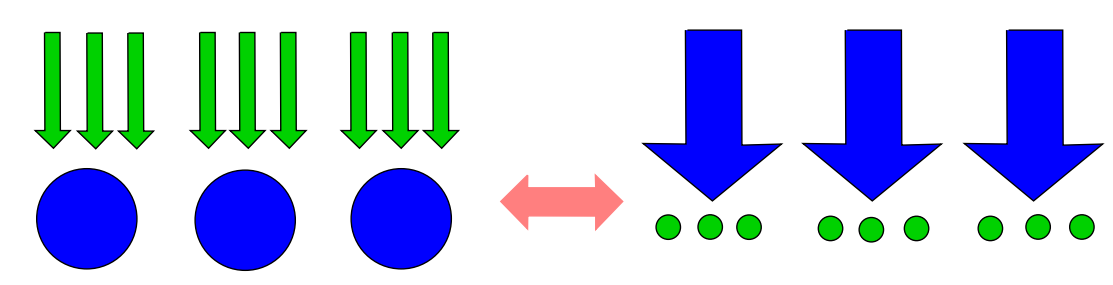

Figure 3. The charge-flux duality in the case of $m=3$. The left figure represents the original particleflux system, where 3-CS fluxes are attached to each particle. Meanwhile, in the right figure, 3-particles are "attached" to one CS flux. The roles of particles (charges) and fluxes are interchanged in the left and right figures. Then, this transformation is called charge-flux duality.

\subsection{The SUSY Chern-Simons description [46]}

We show how the charge-flux duality is naturally generalized in the SUSY QHE. In the Euclidean super space-time $\mathbb{R}^{3 \mid 2}$, there exist the super matter currents $J_{a}, J_{\alpha}$ and the 2-rank super field strengths $F_{a b}, F_{a \alpha}, F_{\alpha \beta}$. The super field strengths are related to each other by the SUSY transformations generated by $Q=L_{\alpha} \xi_{\alpha}$ :

$$
\begin{aligned}
& \delta_{\xi} F_{a b}=-\frac{1}{2} F_{a \alpha}\left(C \sigma_{b} \xi\right)_{\alpha}+\frac{1}{2}\left(C \sigma_{a} \xi\right)_{\alpha}, \quad \delta_{\xi} F_{a \alpha}=\frac{1}{2} F_{a b}\left(\sigma_{b} \xi\right)_{\alpha}+\frac{1}{2} F_{\alpha \beta}\left(C \sigma_{a} \xi\right)_{\beta}, \\
& \delta_{\xi} F_{\alpha \beta}=-\frac{1}{2} F_{a \alpha}\left(\sigma_{b} \xi\right)_{\beta}-\frac{1}{2} F_{a \beta}\left(\sigma_{a} \xi\right)_{\alpha} .
\end{aligned}
$$

Since the number of components of the super-vector currents $(=5)$ and that of 2-rank super tensor field strengths $(=12)$ do not match, one may suspect whether the charge-flux duality exists in the SUSY case. However, 12-dimensional 2-rank tensors are irreducibly decomposed to $\mathbf{5} \oplus \mathbf{7}$. The $\mathbf{5}$-dimensional field strengths, which we call the super-vector field strengths, are explicitly constructed as

$$
F_{a} \equiv \frac{1}{2} \epsilon_{a b c} F_{b c}+i \frac{1}{4}\left(C \sigma_{a}\right)_{\alpha \beta} F_{\alpha \beta}, \quad F_{\alpha} \equiv-i \frac{1}{2}\left(C \sigma_{a}\right)_{\alpha \beta} F_{a \beta} .
$$

Indeed, under the $O S p(1 \mid 2)$ SUSY transformation, they form a multiplet:

$$
\delta_{\xi} F_{a}=\frac{1}{2} F_{\alpha}\left(\sigma_{a} \xi\right)_{\alpha}, \quad \delta_{\xi} F_{\alpha}=\frac{1}{2} F_{a}\left(C \sigma_{a} \xi\right)_{\alpha} .
$$

With the super-vector field strengths, it is possible to develop the charge-flux duality even in the SUSY case. There exists one-to-one correspondence between the matter currents and the super-vector field strengths,

$$
J_{a} \leftrightarrow F_{a}, \quad J_{\alpha} \leftrightarrow F_{\alpha},
$$

and the charge conservation is consistently transfered to the Bianchi identity again: $\partial_{a} J_{a}+$ $\partial_{\alpha} J_{\alpha}=0 \leftrightarrow \partial_{a} F_{a}+\partial_{\alpha} F_{\alpha}=0$. Taking the inner product between $\left(A_{a}, A_{\alpha}\right)$ and $\left(F_{a}, F_{\alpha}\right)$, our SUSY Chern-Simons Lagrangian ${ }^{11}$ is constructed as

$$
\mathcal{L}_{s C S}=F_{a} A_{a}+F_{\alpha} A_{\alpha}=\epsilon_{a b c} A_{a} \partial_{b} A_{c}-i\left(C \sigma_{a}\right)_{\alpha \beta} A_{\alpha} \partial_{a} A_{\beta}+2 i\left(C \sigma_{a}\right)_{\alpha \beta} A_{\alpha} \partial_{\beta} A_{a} .
$$

The SUSY Chern-Simons Lagrangian (6.7) possesses the apparent $O S p(1 \mid 2)$ global symmetry and the $U(1)$ gauge invariance up to total derivatives:

$$
\begin{aligned}
\delta \mathcal{L}_{s C S} & =\partial_{a}\left(\Lambda F_{a}\right)+\partial_{\alpha}\left(\Lambda F_{\alpha}\right) \\
& =\frac{1}{2} \partial_{a}\left(\Lambda \epsilon_{a b c} F_{b c}\right)-i \frac{1}{2} \partial_{\alpha}\left(\Lambda\left(C \sigma_{a}\right)_{\alpha \beta} F_{a \beta}\right)+i \frac{1}{4} \partial_{a}\left(\Lambda\left(C \sigma_{a}\right)_{\alpha \beta} F_{\alpha \beta}\right),
\end{aligned}
$$

\footnotetext{
${ }^{11}$ There are various types of SUSY CS theories, for instance [47, 48, 49]. Here, we develop a new type of SUSY CS theory that is defined on supermanifold. The matrix version of (6.7) plays a crucial role for realization of fuzzy supersphere in supermatrix model [16].
} 
where $\Lambda$ is the $U(1)$ gauge parameter. It is also possible to show that our SUSY CS Lagrangian possesses topological properties analogous to the original Chern-Simons theory; it exhibits SUSY linking number, SUSY topological mass generations and etc [46]. With this SUSY CS term, the Chern-Simons-Landau-Ginzburg (CSLG) Lagrangian is constructed as

$$
\mathcal{L}_{C S L G}=A_{a} J_{a}+A_{\alpha} J_{\alpha}+\frac{1}{4 m \pi}\left(F_{a} A_{a}+F_{\alpha} A_{\alpha}\right)+\cdots,
$$

where $\cdots$ includes the kinetic term of matter field, the Coulomb potential energy and etc. Substituting the dual CS fields for the matter currents, the SUSY CS Lagrangian with interaction term is expressed as

$$
\mathcal{L}=\mathcal{L}_{I}+\mathcal{L}_{s C S}=\left(A_{a} \tilde{F}_{a}+A_{\alpha} \tilde{F}_{\alpha}\right)+\frac{1}{4 m \pi}\left(A_{a} F_{a}+A_{\alpha} F_{\alpha}\right) .
$$

Taking advantage of the duality, the dual CSLG Lagrangian is systematically derived [46]. For instance, integrating out the original CS fields $\left(A_{a}, A_{\alpha}\right)$ in equation (6.9), we obtain the dual CS Lagrangian:

$$
\begin{aligned}
\tilde{\mathcal{L}}_{s C S} & =-m \pi\left(\tilde{A}_{a} \tilde{F}_{a}+\tilde{A}_{\alpha} \tilde{F}_{\alpha}\right) \\
& =-\frac{m \pi}{2}\left(\epsilon_{a b c} \tilde{A}_{a} \tilde{F}_{b c}-i\left(C \sigma_{a}\right)_{\alpha \beta} \tilde{A}_{\alpha} \tilde{F}_{a \beta}+\frac{i}{2}\left(C \sigma_{a}\right)_{\alpha \beta} \tilde{A}_{a} \tilde{F}_{\alpha \beta}\right) .
\end{aligned}
$$

The dual CS Lagrangian is identical to the original SUSY CS Lagrangian (6.8) except for the inverse CS coupling, as found in the original bosonic case. In a low energy limit, the dual CSLG Lagrangian takes the form

$$
L_{\mathrm{eff}}=2 \pi \sum_{p}\left(\dot{x}_{i}^{p} \tilde{A}_{i}+\dot{\theta}_{\alpha}^{p} \tilde{A}_{\alpha}\right)-V+\tilde{\mathcal{L}}_{s C S}
$$

where $x_{i}^{p}$ and $\theta_{\alpha}^{p}$ denote the position of the $p$-th vortex on the superplane, and $V$ denotes the Coulomb potential term. From $L_{\text {eff }}$, the equation of motion for vortex is derived as

$$
2 \pi\left(-\tilde{F}_{i j} \dot{x}_{j}^{p}+\tilde{F}_{i \alpha} \dot{\theta}_{\alpha}^{p}\right)=E_{i}, \quad 2 \pi\left(\tilde{F}_{i \alpha} \dot{x}_{i}^{p}+\tilde{F}_{\alpha \beta} \dot{\theta}_{\beta}^{p}\right)=C_{\alpha \beta} E_{\beta} .
$$

Equations (6.10) suggest that the vortex moves perpendicularly to the direction of the applied super electric fields:

$$
E_{i} \dot{x}_{i}^{p}+C_{\alpha \beta} E_{\alpha} \dot{\theta}_{\beta}^{p}=0
$$

which manifests the Hall orthogonality in the SUSY sense.

\section{Summary and discussion}

We overviewed the developments of the SUSY QHE. It was shown that the framework of QHE was naturally supersymmetrized based on the SUSY Hopf map. In the construction of the SUSY QHE we have encountered many exotic mathematical and physical ideas. The SUSY Hopf fibration was crucial in construction of the spherical SUSY QHE. In the LLL limit, the fuzzy supersphere naturally emerges. In the planar SUSY QHE, we explored the SUSY Landau problem, and found the existence of $\mathcal{N}=2$ SUSY. (The existence of the $\mathcal{N}=2$ SUSY may be a generic feature of SUSY planar Landau models [24, 25].) With appropriate interpretation of the Grassmann quantity, we have shown that the SUSY Laughlin wavefunction contains the original Laughlin and the Moore-Read states as its first and last component wavefunctions. A SUSY CS field theory is also developed as the appropriate effective field theory for the SUSY 
QHE. The newly derived Chern-Simons theory is invariant under the global $O S p(1 \mid 2)$ and local $U(1)$ transformations, and shares topological features with the original CS theory. The Hall orthogonality and the charge-flux duality are consistently generalized in the SUSY framework.

However, there still remain many issues to be addressed within the formulation of the SUSY QHE, such as edge excitations, hydrodynamic description and relations to integrable systems. Among them, one of the most important issues is to explore applications to real condensed matter physics. As we have seen, the SUSY brings a unified picture of the original Laughlin and the Moore-Read states. It would be worthwhile to speculate what insights such unification could yield to the original QHE. Though the SUSY QHE provides a concrete physical realization of the non-anti-commutative geometry, our set-up is still restricted to low dimensions. It is quite tempting to extend our SUSY formulation to higher dimensions. The construction of higher dimensional SUSY QHE may be beneficial to the understanding of higher dimensional fuzzy super geometries, in particular classical solutions of supermatrix models. Besides, as reported in [50, 51], QHE contains mathematical structures similar to the twistor theory. It is also interesting to exploit relations between the SUSY QHE and supertwistor theory. Further developments of QHE may bring fruitful consequences in a wide realm of modern physics.

\section{Acknowledgements}

I would like to thank Yusuke Kimura for collaborations in early stage of this work.

\section{References}

[1] Ezawa Z.F., Tsitsishvili G., Hasebe K., Noncommutative geometry, extended $W_{\infty}$ algebra and Grassmannian solitons in multicomponent quantum Hall systems, Phys. Rev. B 67 (2003), 125314, 16 pages, hep-th/0209198.

[2] Zhang S.C., Hu J.P., A four dimensional generalization of the quantum Hall effect, Science 294 (2001), 823-828, cond-mat/0110572.

[3] Karabali D., Nair V.P., Quantum Hall effect in higher dimensions, Nuclear Phys. B 641 (2002), 533-546, hep-th/0203264.

[4] Bernevig B.A., Hu J.P., Toumbas N., Zhang S.C., The eight dimensional quantum Hall effect and the octonions, Phys. Rev. Lett. 91 (2003), 236803, 4 pages, cond-mat/0306045.

[5] Hasebe K., Kimura Y., Dimensional hierarchy in quantum Hall effects on fuzzy spheres, Phys. Lett. B 602 (2004), 255-260, hep-th/0310274.

[6] Nair V.P., Randjbar-Daemi S., Quantum Hall effect on $S^{3}$, edge states and fuzzy $S^{3} / \mathbf{Z}_{2}$, Nuclear Phys. B 679 (2004), 447-463, hep-th/0309212.

[7] Jellal A., Quantum Hall effect on higher dimensional spaces, Nuclear Phys. B 725 (2005), 554-576, hep-th/0505095.

[8] Daoud M., Jellal A., Quantum Hall effect on the flag manifold $F_{2}$, hep-th/0610157.

[9] Landi G., Spin-Hall effect with quantum group symmetry, Lett. Math. Phys. 75 (2006), 187-200, hep-th/0504092.

[10] Karabali D., Nair V.P., Quantum Hall effect in higher dimensions, matrix models and fuzzy geometry, J. Phys. A: Math. Gen. 39 (2006), 12735-12763, hep-th/0606161.

[11] Murakami Sh., Nagaosa N., Zhang S.C., Dissipationless quantum spin current at room temperature, Science 301 (2003), 1348-1351, cond-mat/0308167.

[12] de Boer J., Grassi P.A., van Nieuwenhuizen P., Non-commutative superspace from string theory, Phys. Lett. B 574 (2003), 98-104, hep-th/0302078.

[13] Ooguri H., Vafa C., The C-deformation of gluino and non-planar diagrams, Adv. Theor. Math. Phys. 7 (2003), 53-85, hep-th/0302109.

[14] Seiberg N., Noncommutative superspace, $N=1 / 2$ supersymmetry, field theory and string theory, J. High Energy Phys. 2003 (2003), no. 6, 010, 17 pages, hep-th/0305248. 
[15] Azuma T., Iso S., Kawai H., Ohwashi Y., Supermatrix models, Nuclear Phys. B 610 (2001), 251-279, hep-th/0102168.

[16] Iso S., Umetsu H., Gauge theory on noncommutative supersphere from supermatrix model, Phys. Rev. D 69 (2004), 105003, 7 pages, hep-th/0311005.

Iso S., Umetsu H., Note on gauge theory on fuzzy supersphere, Phys. Rev. D 69 (2004), 105014, 7 pages, hep-th/0312307.

[17] Balachandran A.P., Kurkcuoglu S., Rojas E., The star product on the fuzzy supersphere, J. High Energy Phys. 2002 (2002), no. 7, 056, 22 pages, hep-th/0204170.

[18] Balachandran A.P., Pinzul A., Qureshi B., SUSY anomalies break $N=2$ to $N=1$ : the supersphere and the fuzzy supersphere, J. High Energy Phys. 2005 (2005), no. 12, 002, 14 pages, hep-th/0506037.

[19] Kurkcuoglu S., Non-linear sigma model on the fuzzy supersphere, J. High Energy Phys. 2004 (2004), no. 3, 062, 12 pages, hep-th/0311031.

[20] Schunck A.F., Wainwright Ch., A geometric approach to scalar field theories on the supersphere, J. Math. Phys. 46 (2005), 033511, 34 pages, hep-th/0409257.

[21] Panero M., Quantum field theory in a non-commutative space: theoretical predictions and numerical results on the fuzzy sphere, SIGMA 2 (2006), 081, 14 pages, hep-th/0609205 (and references therein).

[22] Ivanov E., Mezincescu L., Townsend P.K., Fuzzy $C P(n \mid m)$ as a quantum superspace, hep-th/0311159.

[23] Ivanov E., Mezincescu L., Townsend P.K., A super-flag Landau model, hep-th/0404108.

[24] Ivanov E., Mezincescu L., Townsend P.K., Planar super-Landau models, J. High Energy Phys. 2006 (2006), no. 1, 143, 23 pages, hep-th/0510019.

[25] Curtright T., Ivanov E., Mezincescu L., Townsend P.K., Planar super-Landau models revisited, J. High Energy Phys. 2007 (2007), no. 4, 020, 25 pages, hep-th/0612300.

[26] Bellucci S., Beylin A., Krivonos S., Nersessian A., Orazi E., $N=4$ supersymmetric mechanics with nonlinear chiral supermultiplet, Phys. Lett. B 616 (2005), 228-232, hep-th/0503244.

[27] Gates S.J. Jr., Jellal A., Saidi E.H., Schreiber M., Supersymmetric embedding of the quantum Hall matrix model, J. High Energy Phys. 2004 (2004), no. 11, 075, 29 pages, hep-th/0410070.

[28] Yu M., Zhang X., Supersymmetric Hamiltonian approach to edge excitations in $\nu=5 / 2$ fractional quantum Hall effect, arXiv:0706.1338.

[29] Haldane F.D.M., Fractional quantization of the Hall effect: a hierarchy of incompressible quantum fluid states, Phys. Rev. Lett. 51 (1983), 605-608.

[30] Frappat L., Sciarrino A., Sorba P., Dictionary on Lie algebras and superalgebras, Academic Press, San Diego, 2000 (and references therein).

[31] Nakahara M., Geometry, topology and physics, IOP Publishing, Bristol, 2003.

[32] Bartocci C., Bruzzo U., Landi G., Chern-Simons forms on principal superfiber bundles, J. Math. Phys. 31 (1987), 45-54.

[33] Landi G., Projective modules of finite type over the supersphere $S^{2,2}$, Differential Geom. Appl. 14 (2001), 95-111, math-ph/9907020.

[34] Hasebe K., Kimura Y., Fuzzy supersphere and supermonopole, Nuclear Phys. B 709 (2005), 94-114, hep-th/0409230.

[35] Hasebe K., Supersymmetric quantum Hall effect on a fuzzy supersphere, Phys. Rev. Lett. 94 (2005), 206802, 4 pages, hep-th/0411137.

[36] Grosse H., Klimcik C., Presnajder P., Field theory on a supersymmetric lattice, Comm. Math. Phys. 185 (1997), 155-175, hep-th/9507074.

[37] Grosse H., Reiter G., The fuzzy supersphere, J. Geom. Phys. 28 (1998), 349-383, math-ph/9804013.

[38] Balachandran A.P., Kurkcuoglu S., Vaidya S., Lectures on fuzzy and fuzzy SUSY physics, hep-th/0511114.

[39] Hatsuda M., Iso S., Umetsu H., Noncommutative superspace, supermatrix and lowest Landau level, Nuclear Phys. B 671 (2003), 217-242, hep-th/0306251.

[40] Hasebe K., Quantum Hall liquid on a noncommutative superplane, Phys. Rev. D 72 (2005), 105017, 9 pages, hep-th/0503162.

[41] Hasebe K., Unification of Laughlin and Moore-Read states in SUSY quantum Hall effect, Phys. Lett. A 372 (2008), 1516-1520, arXiv:0705.4527. 
[42] Moore G., Read N., Nonabelions in the fractional quantum hall effect, Nuclear Phys. B 360 (1991), 362-396.

[43] Greiter M., Wen X-G., Wilczek F., Paired Hall state at half filling, Phys. Rev. Lett. 66 (1991), 3205-3208.

[44] Zhang S.C., Hansson T.H., Kivelson S., Effective-field-theory model for the fractional quantum Hall effect, Phys. Rev. Lett. 62 (1989), 82-85.

[45] Lee D.H., Fisher M.P.A., Anyon superconductivity and charge-vortex duality. Fractional statistics in action. Internat. J. Modern Phys. B 5 (1991), 2675-2699 (for instance).

[46] Hasebe K., Supersymmetric Chern-Simons theory and supersymmetric quantum Hall liquid, Phys. Rev. D 74 (2006), 045026, 12 pages, hep-th/0606007.

[47] Nissimov E., Pacheva S., Phase transition and $1 / N$ expansion in $(2+1)$-dimensional supersymmetric sigma models, Lett. Math. Phys. 5 (1981), 67-73.

Nissimov E., Pacheva S., Parity-violating anomalies in supersymmetric gauge theories, Phys. Lett. B 155 (1985), 76-82.

Nissimov E., Pacheva S., Anomalous generation of Chern-Simons terms in $D=3, N=2$ supersymmetric gauge theories, Lett. Math. Phys. 11 (1986), 43-49.

[48] Lee B.H., Lee C.K., Min H., Supersymmetric Chern-Simons vortex systems and fermion zero modes, Phys. Rev. D 45 (1992), 4588-4599.

[49] Ezawa K., Ishikawa A., $O s p(1 \mid 2)$ Chern-Simons gauge theory as $2 \mathrm{D} N=1$ induced supergravity, Phys. Rev. D 56 (1997), 2362-2368, hep-th/9612031.

[50] Sparling G., Twistor theory and the four-dimensional quantum Hall effect of $\mathrm{Zhang}$ and $\mathrm{Hu}$, cond-mat/0211679.

[51] Mihai D., Sparling G., Tillman Ph., Non-commutative time, the quantum Hall effect and twistor theory, cond-mat/0401224. 